\title{
Extension of the Stoichiometric Calibration of CT Hounsfield values to Metallic Materials
}

https://doi.org/10.1515/cdbme-2020-3135

\begin{abstract}
Nowadays, patients with metallic implants undergoing radiotherapy may suffer from inaccuracy in the treatment plan caused by the implant. To ensure a precise plan an accurate relation between Hounsfield values of the computer tomographic (CT) images and the electron density of the elements and material mixtures is indispensable. In order to extend the stoichiometric calibration approach known for tissues to the regime of metallic materials, the basic physical equations as well as approximations in the parametrization and fitting are carefully reviewed. CT images of a standard calibration phantom and pure metallic samples up to the atomic number $Z=29$ were acquired for various energies. Hounsfield values were determined on an extended Hounsfield scale which allows the mapping of material having high atomic number $Z$. It is found that from basic physics an empirical factorization of the cross-sections into a function of $Z$ and a function of photon energy $E$ is not allowed over a wide range of $Z$. Specifically, the parameterization for tissue like materials cannot be prolonged to materials with high $-Z$. Thus, the calibration is subdivided into regions of materials and its accuracy is quantified in each region. It depends, among others, on the knowledge of the $\mathrm{X}$-ray photon spectra, the segmentation of the material samples and the empirical parameterization of the linear-attenuation coefficient.
\end{abstract}

Keywords: computed tomography, calibration, extended Hounsfield Units, stoichiometric calibration, electron density, radio therapy

\section{Introduction}

The accuracy of radiotherapy treatment of patients with metallic implants is still challenging as treatment plans rely on the accuracy of the electron density $\rho^{e}$ of the tissue or implant ma-

\footnotetext{
${ }^{*}$ Corresponding author: Zehra Ese, Department of Electrical Engineering and Applied Natural Science, Westphalian University, Campus Gelsenkirchen, Germany, and Department of General and Theoretical Electrical Engineering, University of Duisburg-Essen and CENIDE - Center of Nanointegration Duisburg-Essen, Bismarckstr. 81, 47048 Duisburg, Germany, e-mail: zehra.ese@stud.uni-due.de

Waldemar Zylka, Department of Electrical Engineering and Applied Natural Science, Westphalian University, Campus Gelsenkirchen, Germany
}

terial. Typically, materials are mixtures of chemical elements and $\rho^{e}$ must be determined using the stoichiometric or another approach [7].

In a clinical environment, CT images, particularly Hounsfield numbers representing the material or tissue, are used to obtain $\rho^{e}$. The majority of commercial CT machines implement a conventional 12-bit scale which is suitable for soft tissue and bones. For metallic materials the use of an extended scale, e.g. 16-bit, is highly beneficial as it maps high- $Z$ materials, like metals, without an ambitious failure [1-3].

A stoichiometric calibration is usually based on the factorization of the cross-section in terms of functions of $Z$ and $E$. This, however, conflicts with rigorous basic physics $a b$ initio calculations rendering such factorization impossible, at least inaccurate [5]. Since the exact physics formulas are hard to compute, particularly for material compounds and polychromatic X-ray distribution, an empirical parameterization procedure based on factorization is used for tissue like materials in the (narrow) diagnostic energy range $E=80-140 \mathrm{keV}$. This approach is very convenient as the coefficients can be fitted to measured CT numbers, thus coping with (vendor and scanner dependent) spectral X-ray energy distribution. Once fitted to a calibration-material set, one is able to predict the electron density or CT numbers of other materials.

In this work, we apply the stoichiometric calibration used for biological tissues to mixtures of $Z \leq 29$ materials, thus, potentially violating some of its assumptions. One objective is to systematically review all assumptions imposed on the stoichiometric calibration used in the clinical environment. The results of a standard calibration procedure for $E=80 \mathrm{kV}$ and $E=120 \mathrm{kV}$ are presented in detail, the validity and accuracy are investigated for high $Z$ materials.

\section{Methods and Materials}

Extended Hounsfield scale. In CT imaging the spatial distribution of the linear x-ray attenuation coefficients $\mu$ representing the tissues is reconstructed into a matrix of voxels. The attenuation coefficient $\mu$ of a particular image voxel is expressed in (Hounsfield or) CT numbers $H$ using the linear function

$$
H(u)=s u+i,
$$

where $u=1000 \mu / \mu_{w}$ is dimensionless with $\mu_{w}$ being the attenuation coefficient of water, $s$ is the slope and $i$ the intercept. 
We denote the unit of $H, s$ and $i$ by HU. Note, that $s, i$ and $u$ are stored in the DICOM file. Equation (1) can be used to represent various scales, e.g. setting $s=1$ and $i=-1000$ the traditional Hounsfield definition is found. Often CT scanners use 12-bit when storing CT measurements. It has been found in $[1,2]$ that with this scale metallic materials provoke errors in dose calculations since they are incorrectly mapped to scale's maximum. An extended scale, e.g. 16-bit, allows correct mapping [2].

$\mathrm{X}$-ray attenuation-coefficient and electron density. The linear attenuation coefficient of a mixture of $M$ chemical elements can be expressed, as a function of energy $E$, by:

$$
\mu\left(E, Z_{i}\right)=\rho^{e} \sum_{i=1}^{M} \frac{\rho_{i}^{e}}{\rho^{e}} \sigma_{i}^{e}\left(E, Z_{i}\right),
$$

where $\rho_{i}^{e}=\rho N_{A} w_{i} Z_{i} / A_{i}$ is the volume electron density (electrons per unit volume) of the $i$-th element and $\rho^{e}=\sum_{i} \rho_{i}$ the total electron density of the mixture (here and in the following the $i$-summation runs from 1 to $M$ ). The mass density of the mixture is $\rho, N_{A}$ is Avogadro's number, $\sigma_{i}^{e}, Z_{i}$ and $A_{i}$ are, respectively, the total scattering cross-section per electron, the atomic number and atomic weight of the $i-$ th element and $w_{i}$ is its proportion by weight. For pure elements $M=w_{i}=1$. The total cross-section per atom is $\sigma_{i}=Z_{i} \sigma_{i}^{e}$.

A CT system must at least be described as a polychromatic beam of photons (potentially) undergoing filtration before crossing the material and being detected by an energy sensitive detector. As in a clinical setting, we assume the X-ray spectrum $S(E)$ to be unknown, but normalized (to 1); for single energy beams $S(E)=\delta\left(E-E^{\prime}\right)$ holds. All monochromatic quantities in (2) must be averaged over $S(E)$ and will be denoted by a hat $(\uparrow)$, e.g.

$$
\widehat{\mu}\left(S, Z_{i}\right)=\int_{0}^{E_{\max }} d E S(E) \mu\left(E, Z_{i}\right) .
$$

While passing an object the beam of a polychromatic spectrum is shifted to higher energies since the cross-sections $\sigma^{e}\left(E, Z_{i}\right)$ are larger for low-energy photons. This beam-hardening effect is position dependent and particularly prominent for high$Z$ materials. In other words, if beam-hardening is not accounted for, the spectrum must be seen as dependent on position $\vec{r}$ in the material, i.e. $S(E, \vec{r})$.

The total X-ray cross-section per atom $\sigma_{i}$ in the energy range $80-140 \mathrm{keV}$ is a sum of cross-sections of three physical processes: photoelectric absorption, $\sigma^{p h}(E, Z)$, coherent (Rayleigh) scattering, $\sigma^{c o h}(E, Z)$, and incoherent (Compton) scattering, $\sigma^{i n c o h}(E, Z)$. For monochromatic beams exact formula for each of these cross-sections were calculated from fundamental physics and found that neither a cross-section nor their sum factorize into a function $K(E)$ and a function $F(Z)$
[5]. Specifically, none of the cross-section can be written as $\sigma^{\circ}\left(E, Z_{i}\right)=K^{\circ}(E) F^{\circ}\left(Z_{i}\right)$, where $\circ$ denotes one of the physical processes mentioned above. Such factorization, however, is (frequently) assumed to hold as it is very beneficial when calculating $\widehat{\mu}\left(S, Z_{i}\right)$.

By restricting $Z$ to the vicinity of a reference element in a mixture, e.g. carbon, a (potentially non-integer) Taylor expansion of $F^{\circ}\left(Z_{i}\right)$ is admissible at some level of accuracy $[5,6,9]$. Thus, by utilizing (3), $\widehat{\mu}\left(S, Z_{i}\right)$ can be parameterized in $Z$ direction by spectrum dependent expansion coefficients $\widehat{K}^{\circ}(S)$. The primary benefit of a parameterization scheme is that its coefficients could be obtained by a least square regression (fit) to Hounsfield values of calibration materials measured with a particular spectrum $S(E)$. This allows a subsequent prediction of CT numbers and electron densities of new materials for the particular energy spectrum.

Simplifying the Taylor expansion in $Z$ to one single power law, i.e. $F^{\circ}\left(Z_{i}\right) \propto Z_{i}^{s}$, one recovers the parameterization originally introduced in [4] for elements near to oxygen and for spectra of the EMI scanner at $60 \mathrm{keV}$ and $80 \mathrm{keV}$ :

$$
\sigma_{i}^{e}\left(E, Z_{i}\right)=K^{p h} Z_{i}^{p-1}+K^{c o h} Z_{i}^{q-1}+K^{i n c o h} Z_{i}^{r-1} .
$$

The coefficients $K^{\circ}$ depend on energy only, $K^{\circ}=K^{\circ}(E)$, the non-integer exponents $p=4.62, q=2.86$ were obtained from fitting to tabulated cross-sections and $r=1$ originates from Compton scattering physics at $E \gg 1 \mathrm{MeV}$. With the aid of (2)-(4) it follows:

$$
\widehat{\mu}\left(S, Z_{i}\right)=\rho^{e}\left(\widehat{K}^{p h} \widetilde{Z}^{p-1}+\widehat{K}^{c o h} \widetilde{Z}^{q-1}+\widehat{K}^{i n c o h} \widetilde{Z}^{r-1}\right),
$$

where $\widetilde{Z}=\left(\sum_{i}\left(\rho_{i}^{e} / \rho^{e}\right) Z_{i}^{s}\right)^{1 / s}$ is a weighted (effective) atomic number. The coefficients $\widehat{K}^{\circ}$ are functions of the spectrum, i.e. $\widehat{K}^{\circ}=\widehat{K}^{\circ}(S)$. In practice, each scanning protocol uses its own energy spectrum and algorithms, potentially including additional corrections, e.g. during reconstruction, which affect the energy spectrum and are included in $S(E)$ in this paper.

The parameterization coefficients $\widehat{K}^{\circ}(S)$ must be determined for the particular $S(E)$ by a fitting procedure to Hounsfield values, via (1), of calibration materials measured with a calibration phantom (with known densities and elemental compositions). Among others, the least square regression procedure in [7] and [8] can be used. The former is a three parameter polynomial fit to (5) with $\widehat{K}^{\circ}$ normalized to the (a priori known) attenuation coefficient $\widehat{\mu}_{w}$ of water. In the approach from [8], which was used in this investigation, $\widehat{K}^{\circ}$ of the calibration material are normalized by $\widehat{K}_{w}^{i n c o h}$ of water forming $\widehat{k}^{\circ}(S)=\widehat{K}^{\circ} / \widehat{K}_{w}^{i n c o h}$ to be obtained from a non-linear fit to

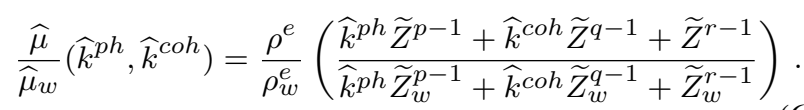


Note that this implies $\widehat{K}^{\circ} \approx \widehat{K}_{w}^{\circ}$ which might be inaccurate for elements or mixtures with atomic number or electron density well away from that of water. Once the scanner-specific coefficients $\widehat{k}^{\circ}$ are known for each energy spectrum, the values of the relative electron density $\rho_{\text {rel }}=\rho^{e} / \rho_{w}^{e}$ of other materials and mixtures with known elemental composition can be computed and look-up-tables for X-ray radiotherapy created using (6).

We employed a tissue characterization phantom model 467 (Gammex) phantom containing known tissue equivalent materials in cylindrical design $(d=28 \mathrm{~mm}, h=70 \mathrm{~mm})$ which was scanned using $80 \mathrm{keV}$ and $120 \mathrm{keV}$ spectra of the Siemens Somatom Force scanner. Additionally, four metallic coin-shaped samples provided by umicore $\AA(d=20 \mathrm{~mm}$, $h=5 \mathrm{~mm})$ from $\mathrm{Al}(Z=13), \mathrm{Cr}(Z=24), \mathrm{Ti}(Z=22), \mathrm{Cu}$ $(Z=29)$ were scanned being in a self-constructed cubic water phantom $(h=350 \mathrm{~mm})$ made of polystyrene material. The samples were fixed at a water equivalent plate made from RW3 (PTW Freiburg GmbH) and submerged in the water phantom to make sure, that the samples are fully covered by water. The samples were positioned in a water depth of $3 \mathrm{~cm}$, measured from the sample surfaces to the waterline. All materials were subdivided into two segments separated by a relative electron density $\rho_{r e l}^{s}$. The least square fit was separately done in the $\rho_{\text {rel }} \leq \rho_{\text {rel }}^{s}$ and in the $\rho_{\text {rel }}>\rho_{\text {rel }}^{s}$ region. We used $\rho_{\text {rel }}^{s}=1.0$ and $\rho_{\text {rel }}^{s}=1.69$ representing water and SB3 cortical bone, respectively.

\section{Results}

At $80 \mathrm{kV}$ and $120 \mathrm{kV}$, respectively, the following mean CT numbers $H_{m}$ are determined from images: $\mathrm{Al} 2587 \mathrm{HU}$ and $2071 \mathrm{HU}$, for Ti $8186 \mathrm{HU}$ and $7273 \mathrm{HU}$, for $\mathrm{Cr} 9263 \mathrm{HU}$ and $9085 \mathrm{HU}$ and for $\mathrm{Cu} 10916 \mathrm{HU}$ and $12168 \mathrm{HU}$. The effect of decreasing CT numbers with increasing tube voltage can be observed for almost all metal samples. The expression of cupping artifacts becomes stronger with increasing tube voltage, as well as with increasing atomic number $Z$, mainly due to beam hardening effects [1-3]. Since, we use a ROI of size $1.8 \mathrm{~mm} \times 6.6 \mathrm{~mm}$ at the central part of a material to determine the mean CT number $H_{m}$ and its error $\sigma_{e r r}$.

The $\widehat{k}^{\circ}$ values were calculated segmentally for two separation points $\rho_{\text {rel }}^{s}$ given above. Figure 1 shows the relative electron densities $\rho_{\text {rel }}$ along all materials versus the corresponding $\mathrm{CT}$ numbers. Figure 1a vs. Fig. 1c and Fig. 1b vs.Fig. 1d display the comparison of the fits in two regions separated by the separation points $\rho_{\text {rel }}^{s}$ and obtained at $80 \mathrm{kV}$ and $120 \mathrm{kV}$, respectively. In each sub-figure the measured CT numbers and those calculated by the stoichiometric calibration method, i.e. Eq. (6), are displayed. To quantify the error, measured CT numbers were additionally set to $H_{m} \pm \sigma_{e r r}$. The calculated CT number is expected to be within $\left[H_{m}-\sigma_{e r r}\right.$, $\left.H_{m}+\sigma_{\text {err }}\right]$.

For material regions in the range $\rho_{\text {rel }}=3.7-8.0$ a considerable mismatch between measured and calculated CT numbers is observed. This cannot be explained by the statistical errors $\sigma_{e r r}$ and must be seen as a systematic error steaming, e.g. from CT image artefacts and an incorrect parametrisation of the cross-section.

Discrepancies between the calculated and the measured CT numbers due to the choice of the material segments can be found in the region of tissue equivalent materials. This is shown in the inset in Fig. 1 for $\rho_{\text {rel }}=1-2$. While for $\rho_{\text {rel }} \leq \rho_{\text {rel }}^{s}$ the calculated CT numbers are comparable, discrepancies are noted for $\rho_{\text {rel }} \geq \rho_{\text {rel }}^{s}$. For the $\rho_{\text {rel }}^{s}=1$ separation point $\Delta H_{\min }^{80 \mathrm{kV}}=42 \mathrm{HU}, \Delta H_{\max }^{80 k \mathrm{~V}}=630 \mathrm{HU}$ and $\Delta H_{\min }^{120 k V}=44 \mathrm{HU}, \Delta H_{m a x}^{120 k V}=251 \mathrm{HU}$. For $\rho_{r e l}^{s}=$ 1.69 we observed $\Delta H_{\min }^{80 k \mathrm{~V}}=0 \mathrm{HU}, \Delta H_{\max }^{80 k V}=1 \mathrm{HU}$ and $\Delta H_{\min }^{120 k V}=10 \mathrm{HU}, \Delta H_{\max }^{120 k V}=11 \mathrm{HU}$. Separating materials at $\rho_{\text {rel }}^{s}=1$ shows an increasing improvement with increasing energy for the whole material area. In contrast, for $\rho_{\text {rel }}^{s}=1.69$ we observe almost energy independence, especially for tissue equivalent materials.

\section{Discussion and Summary}

While former stoichiometric calibration for relative electron density focused on human tissue substitutes, the present study extends the approach to metallic materials. Moving to high- $Z$ materials requires review of basic physics and approximations traditionally used. Consequently, the parameterization of the cross-sections for metals apparently differs from that for materials nearby human tissue. As metallic materials do not necessarily obey the calibration curve of tissues, the calibration should be done for various material regions. The computation of Hounsfield values of metallic materials from CT images is susceptible to beam hardening artifacts. Even if the statistical error is small, the systematic error due to that artifact requires additional quantification.

The applicability of the stoichiometric calibration based on parametrization from [4] is limited in the presence of metallic materials. Among others, accuracy and robustness relies on the division of materials in segments and the knowledge of the X-ray photon spectra. Specific spectra can, however, be incorporated into the approach. A one power-law parameterization for wide $Z$ ranges remains in conflict with rigourous physics and must be replaced by an improved expression. 


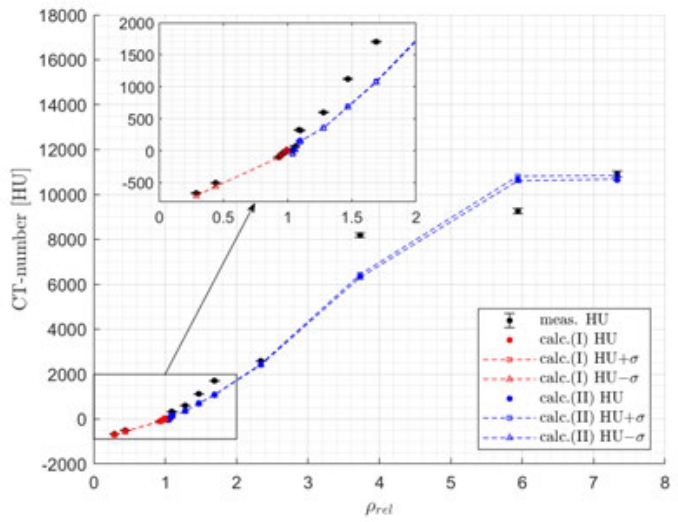

(a) $E=80 \mathrm{kV}$, separation at $\rho_{\text {rel }}^{s}=1$

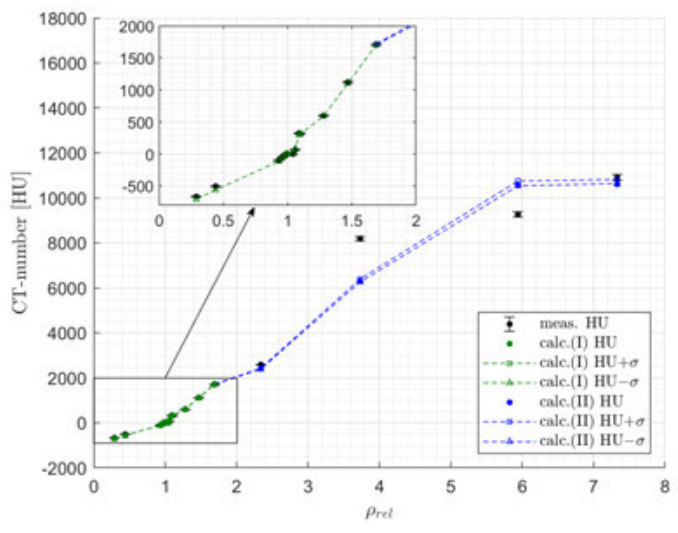

(c) $E=80 \mathrm{kV}$, separation at $\rho_{\text {rel }}^{s}=1.69$

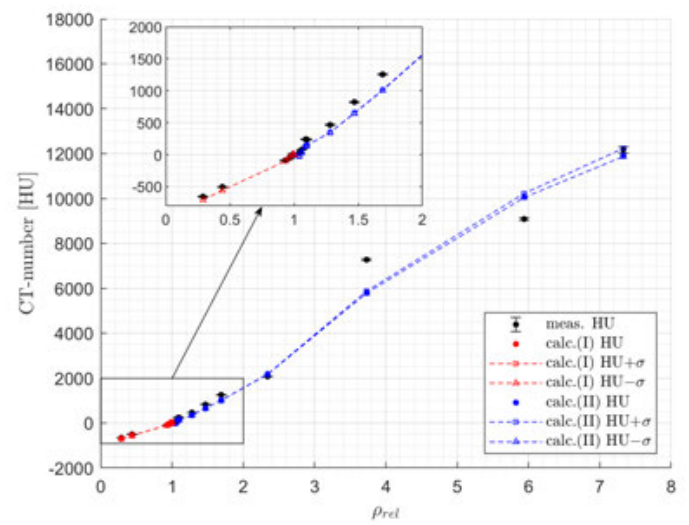

(b) $E=120 \mathrm{kV}$, separation at $\rho_{\text {rel }}^{s}=1$

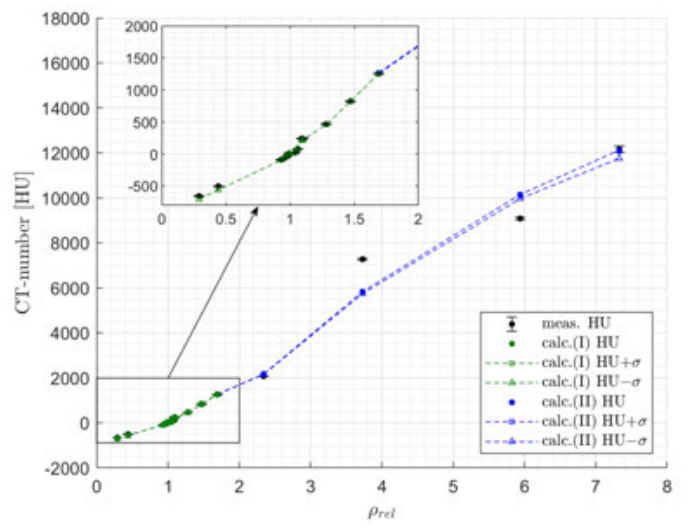

(d) $E=120 \mathrm{kV}$, separation at $\rho_{\text {rel }}^{s}=1.69$

Fig. 1: The relative electron density $\rho_{\text {rel }}$ versus measured and calculated CT numbers at $80 \mathrm{kV}$ and $120 \mathrm{kV}$ for Gammex 467 phantom materials and metals. Measured CT numbers are displayed with the error of the means (bars). The least square fits are shown as dashed lines. At each energy, two different fittings are compared (a) vs (c) and (b) vs (d), they differ in separation point $\rho_{r e l}^{s}$.

\section{Author Statement}

Research funding: The author state no funding involved. Conflict of interest: Authors state no conflict of interest.

\section{References}

[1] Ese Z, Kressmann M, Kreutner J, Schaefers G, Erni D, Zylka W. Influence of conventional and extended CT scale range on quantification of Hounsfield units of medical implants and metallic objects. technisches messen - tm. 2018; 85(5):343350.

[2] Ese Z, Qamhiyeh S, Kreutner J, Schaefers G, Erni D, Zylka W. CT Extended Hounsfield Unit range in radiotherapy treatment planning for patients with implantable medical devices. Springer Nature Singapore IFMBE Proc 2019;68(3),599-603.

[3] Ese Z, Zylka W. Influence of 12-bit and 16-bit CT values of metals on dose calculation in radiotherapy using PRIMO, a Monte Carlo code for clinical linear accelerators, Biomed Eng - Biomed Tech 2019;5(1):597-600.
[4] Rutherford R A, Pullan B R, Isherwood I. Measurement of effective atomic number and electron density using an EMI scanner. Neuroradiology 1976;11(1):15-21.

[5] Jackson D F, Hawkes D J. X-ray attenuation coefficients of elements and mixtures. Elsevier - Physics Reports 1981;70(3):169-233.

[6] $R$ G Ouellet $R G$ and Schreiner $L$ J. A parameterization of the mass attenuation coefficients for elements with $Z=1$ to $Z=92$ in the photon energy range from approximately 1 to $150 \mathrm{keV}$. Physics in Medicine and Biology 1991;36:987-999.

[7] Schneider U, Pedroni E, Lomax A. The calibration of CT Hounsfield units for radiotherapy treatment planning. Physics in Medicine and Biology 1996;41:111-124.

[8] Schneider W, Bortfeld T and Schlegel W. Correlation between CT numbers and tissue parameters needed for Monte Carlo simulations of clinical dose distributions. Physics in Medicine and Biology 2000;45:459-478.

[9] Midgley S M. A parameterization scheme for the x-ray linear attenuation coefficient and energy absorption coefficient. Physics in Medicine and Biology 2004; 49:307-325. 